\title{
Modified Projective Outer Synchronization between Two Fractional Order Complex Networks via Adaptive Control
}

\author{
Hong-juan Liu, ${ }^{1,2}$ Guo-qi Liu, ${ }^{1}$ Hai Yu, ${ }^{1}$ and Zhi-liang Zhu ${ }^{1}$ \\ ${ }^{1}$ Software College, Northeastern University, Shenyang 110819, China \\ ${ }^{2}$ School of Information Science \& Engineering, Northeastern University, Shenyang 110819, China \\ Correspondence should be addressed to Zhi-liang Zhu; zhuzl@swc.neu.edu.cn
}

Received 21 January 2014; Revised 21 March 2014; Accepted 22 March 2014; Published 23 April 2014

Academic Editor: Jinde Cao

Copyright (c) 2014 Hong-juan Liu et al. This is an open access article distributed under the Creative Commons Attribution License, which permits unrestricted use, distribution, and reproduction in any medium, provided the original work is properly cited.

\begin{abstract}
The modified projective outer synchronization between two different fractional order complex dynamical networks with different node dynamics and different topological structures is investigated in this paper. Based on the stability theorem of fractional order systems, the adaptive controllers and sufficient criteria for achieving the synchronization are given by applying the nonlinear control. The proposed method is also applicable to modified projective outer synchronization between two fractional order complex networks with identical topological structures. In addition, there are fewer restrictions on the coupling configuration matrix and inner-coupling matrix. The corresponding numerical results coincide with theoretical analysis, which also show that feedback strength and fractional order can effectively affect the synchronization performance.
\end{abstract}

\section{Introduction}

Since the pioneering work of Pecora and Carroll synchronizing identical chaotic systems with different initial conditions [1], various synchronization scenarios have been studied for chaotic systems [2-4]. In recent years, research on complex networks has attracted increasing attention due to its wide and potential applications in various fields. A complex dynamical network consists of a set of interconnected nodes, which are dynamic systems with specific contents. The network model widely exists in nature and society, for example, the World Wide Web, Internet, social networks, biological neural networks, food webs, proteins, the transportation networks, and so forth [5-8]. With the development of the property and dynamics of complex networks, the chaos synchronization of complex networks has become a research focus owing to its engineering applications in secure communications, neural networks, biological systems, parallel image processing, and so forth. Therefore, much work has been done for the synchronization of complex networks in the literature [9-12].

The above research results have laid a good foundation for further theoretical research and practical application of synchronization dynamics in complex network. However, existing research efforts mostly focused on synchronization of integer order complex networks (ICNs), whose dynamics are described by integer order differential equations. In fact, fractional order systems provide an excellent instrument for the description of memory and hereditary properties of various materials and processes. Moreover, they include traditional integer order derivatives as a special case. It would be much better if many practical problems are described by fractional order dynamical systems rather than integer order ones. On the other hand, due to the limited theories for analyzing the dynamics of fractional order systems, synchronization of fractional order complex systems (FCNs) is still a challenging research topic and studies about it are still few [13-16]. In [13], authors studied the synchronized motions in a star network of coupled fractional order systems in which the major element is coupled to each of the noninteracting individual elements. In [14], Chen et al. discussed cluster synchronization of complex dynamical networks with fractional order dynamical nodes. Only the nodes in one community which have direct connections to the nodes in other communities are needed to be controlled, resulting in 
reduced control cost. In [15], projective synchronization of a complex network is studied and the coupling functions of the connected nodes are identified. In [16], Wong et al. investigated robust synchronization of fractional order complex dynamical networks with parametric uncertainties. Based on the properties of the Kronecker product and the stability of the fractional order system, the robust synchronization criteria are derived by applying the nonlinear control.

The synchronization among the nodes of a single network has been termed "inner synchronization" in the above literatures. In this case, it has been shown that the stability of synchronized states depends on the details of the underlying network topology. In contrast, the synchronization between the corresponding nodes of two coupled networks is called "outer synchronization." In the real world, there are a great many examples about relationships between different networks, such as the original spreading of infectious diseases between two communities, the balance of beneficial bacteria and pathogenic bacteria in our digestive systems, and predator-prey interactions in ecological systems. These examples show that it is necessary and significant to investigate the dynamics between two coupled networks. At present, the outer synchronization between coupled ICNs has been studied extensively [17-19]. However, to the best of our knowledge, few papers investigate the synchronization between two complex networks with fractional order nodes and the only four attempts are the works in [20-23]. By designing nonlinear controllers, $\mathrm{Wu}$ and $\mathrm{Lu}$ first reported outer synchronization phenomena between two coupled FCNs [20]. Also, Asheghan et al. considered robust outer synchronization between two FCNs coupled by an openplus-closed-loop scheme [21]. More recently, authors in [22] address the global outer synchronization problem between two FCNs coupled in a drive-response configuration. In particular, for a given FCN composed of Lur'e systems, an observer-type response network with nonfragile output feedback controllers is constructed. Authors in [23] propose a novel projective outer synchronization between unidirectionally coupled uncertain FCNs through scalar transmitted signals. However, among these references, only the work in [23] considers projective synchronization between two FCNs with identical nodes and identical topological structures. Thus, it is necessary to construct a more general projective synchronization scheme between two coupled FCNs.

In this paper, we discussed the modified projective outer synchronization (MPOS) problem between two FCNs with different node dynamics and different topological structures. By using adaptive control scheme, a nonlinear controller is designed to achieve the MPOS. The controller is also applicable to the MPOS of two FCNs with identical topological structures. Based on the stability theory of fractional order systems, basic criteria in the format of linear matrix inequalities (LMIs) for MPOS are proposed. Finally, the effectiveness and feasibility of the designed control strategy for MPOS are demonstrated by numerical simulations on chaotic drive-response FCNs. These simulations indicate that increasing the fractional order and the feedback strength will accelerate the speed of outer synchronization.

\section{Model Description and Preliminaries}

There are several definitions of fractional derivatives. The Caputo derivative is more popular in the real applications [24], which is defined as

$$
\frac{d^{q} f(t)}{d t^{q}} \equiv D^{q} f(t)=\frac{1}{\Gamma(m-q)} \int_{0}^{t}(t-\tau)^{m-q-1} f^{(m)}(\tau) d \tau,
$$

where $m$ is the smallest integer larger than $q, D^{q}$ denotes the Caputo definition of the fractional derivative, $f^{(m)}(t)$ is the $m$-order derivative in the usual sense, and $\Gamma$ stands for gamma function.

Now, consider a class of complex networks consisting of $N$ identical coupled nodes, in which each node is an $n$ dimensional fractional order chaotic system. The standard derivative is replaced by a fractional derivative as the following:

$$
D^{q} x_{i}(t)=f\left(x_{i}(t)\right)+a \sum_{j=1}^{N} c_{i j} A x_{j}(t), \quad i=1,2, \ldots, N,
$$

where $x_{i} \in R^{n}$ is the state vector of the $i$ th node, $f \in R^{n}$ is a continuous differentiable vector function that describes the dynamics of the individual node, $a>0$ is the coupling strength, $A$ is an inner-coupling matrix satisfying $A>0$, and $C=\left(c_{i j}\right)_{N \times N}$ is the coupling configuration matrix representing the coupling strength and the topological structure of the network, in which $c_{i j}$ is defined as follows: if there is a connection from node $i$ to node $j(j \neq i), c_{i j}>0$; otherwise, $c_{i j}=0(j \neq i)$. The diagonal elements of matrix $C$ are defined as

$$
c_{i i}=-\sum_{j=1, j \neq i}^{N} c_{i j}, \quad i=1,2, \ldots, N .
$$

In order to investigate outer synchronization between two different networks, we consider (2) as the master network and assume the response complex dynamical network containing $N$ dynamical nodes as follows:

$$
\begin{array}{r}
D^{q} y_{i}(t)=g\left(y_{i}(t)\right)+a \sum_{j=1}^{N} d_{i j} A y_{j}(t)+u_{i}(t), \\
i=1,2, \ldots, N,
\end{array}
$$

where $y_{i} \in R^{n}$ is the state vector of the node $i, g \in R^{n}$ is a continuous differentiable vector function that describes the dynamics of the individual node, $a>0$ is the coupling strength, $A$ has the same meaning as that in (2), $D=\left(d_{i j}\right)_{N \times N}$ is the coupling configuration matrix which has the same meaning as matrix $C$, and $u_{i}(t)$ is the controller for node $i$ to be designed according to the specific node dynamics and topological structures of the drive and response networks. Here, the coupling configuration matrices $C$ and $D$ are not necessarily symmetric or irreducible. We also do not impose any restriction on the inner-coupling matrix $A$. 
Next, according to the definition of modified projective synchronization between two chaotic systems, we will give the definition of MPOS between the drive FCN (2) and the response FCN (4).

Definition 1 (MPOS). For the drive network (2) and response network (4), it is said to be modified projective outer synchronization (MPOS) respect to the scaling factor matrix $\alpha=\operatorname{diag}\left(\alpha_{1}, \alpha_{2}, \ldots, \alpha_{N}\right)$, if there exists a controller $u_{i}(t)$, such that

$$
\begin{array}{r}
\lim _{t \rightarrow+\infty}\left\|e_{i}(t)\right\|=\lim _{t \rightarrow+\infty}\left\|y_{i}(t)-\alpha_{i} x_{i}(t)\right\|=0, \\
i=1,2, \ldots, N .
\end{array}
$$

always holds.

Remark 2. For unidirectional coupled FCN (2) and (4), we can see from Definition 1 that MPOS means that the corresponding node state vectors $x_{i}(t)$ and $y_{i}(t)$ could achieve synchronization with respect to the scaling factor $\alpha_{i}$ regardless of synchronization of inner networks. Therefore, the two networks may contain isolated clusters or nodes. The coupling configuration matrix is not necessary to be irreducible.

Remark 3. It is easy to see that arbitrary constant matrix $\alpha$ can be tuned to realize various outer synchronization behaviors between states $x_{i}(t)$ and $y_{i}(t)$. For example, if $\alpha_{1}=\alpha_{2}=$ $\cdots=\alpha_{N}=1$, we can observe complete outer synchronization (COS); when $\alpha_{1}=\alpha_{2}=\cdots=\alpha_{N}=-1$, antiphase out synchronization (AOS) is obtained. Moreover, we can also realize amplification or attenuation of the oscillation in the drive FCN (2) relative to that in the response FCN (4) by choosing $\left|\alpha_{i}\right|>1, i=1,2, \ldots, N$ or $\left|\alpha_{i}\right|<1, i=1,2, \ldots, N$, respectively.

Lemma 4 (see [25]). For a given autonomous fractional order linear system

$$
D^{q} x(t)=f(x)=W x
$$

where $0<q<1, x \in R^{n}$ is a state vector, and $W \in R^{n \times n}$ is a constant matrix, the system is asymptotically stable if and only if $\left|\arg \left(\lambda_{i}\right)\right|>q \pi / 2, i=1,2, \ldots, n$, where $\arg \left(\lambda_{i}\right)$ denotes the argument of the eigenvalue $\lambda_{i}$ of $W$. Also, system (6) is stable if and only if either it is asymptotically stable or those critical eigenvalues which satisfy $\left|\arg \left(\lambda_{i}\right)\right|=q \pi / 2$ have a geometric multiplicity one.

Theorem 5 (see [26]). For a class of autonomous fractional order nonlinear system

$$
D^{q} x(t)=f(x)=W(x) x,
$$

where $0<q<1, x \in R^{n}$ is a state vector, and $W(x) \in$ $R^{n \times n}$ is a continuous coefficient matrix, which contains the state variables. If there exists a real symmetric positive definite matrix $P>0$, such that the equation

$$
J=x^{T} P D^{q} x \leq 0
$$

always holds for any state $x(t)$, then the trivial solution $x=0$ of system (7) is asymptotically stable.

Proof. Obviously, $x=0$ is an equilibrium point of system (7) from $f(x)=W(x) x$. Because $J=x^{T} P D^{q} x \leq 0$, we have $J+J^{T}=x^{T}\left(P W(x)+W^{H}(x) P\right) x \leq 0$. Denoting $P W(x)+$ $W^{H}(x) P=Q(x)$, we can conclude that $P W(0)+W^{H}(0) P=$ $Q(0) \leq 0$ always holds for $x=0$. Otherwise, if $Q(0)>$ 0 , by $\lim _{x \rightarrow 0} Q(x)=Q(0)$, there must exist state $x^{*} \neq 0$, such that matrix $Q\left(x^{*}\right)>0$; thus, we have $x^{* T}\left(P W\left(x^{*}\right)+\right.$ $\left.W^{H}\left(x^{*}\right) P\right) x^{*}=x^{* T} Q\left(x^{*}\right) x^{*}>0$, a contradiction. Therefore, the inequality $P W(0)+W^{H}(0) P=Q(0) \leq 0$ always holds for $x=0$.

Assume that $\lambda$ is one of the eigenvalues of matrix $W(0)$ and the corresponding nonzero eigenvector is $\xi$; that is, $W(0) \xi=\lambda \xi$. Multiply the left of $P W(0)+W^{H}(0) P=Q(0)$ by $\xi^{T}$ and its right by $\xi$. We derive that

$$
\xi^{T}\left(P W(0)+W^{H}(0) P\right) \xi=\xi^{T} Q(0) \xi \leq 0 .
$$

Because $P>0$, we can obtain $\lambda+\bar{\lambda} \leq 0$. Thus, we have $|\arg (\lambda)| \geq \pi / 2>q \pi / 2$ for $0<q<1$. According to the stability theory of fractional-order nonlinear systems in Lemma 4, the trivial solution $x=0$ of $\operatorname{system~(7)~is~}$ asymptotically stable.

The proof is completed.

Assumption 6 (see [11]). For the positive definite matrix $A$ in network (2), there exists a constant matrix $T$ such that

$$
\begin{array}{r}
(x-y)^{T}(f(x)-f(y)) \leq(x-y)^{T} T A(x-y), \\
\forall x, y \in R^{n} .
\end{array}
$$

always holds.

According to Definition 1, the error vectors can be expressed as $e_{i}(t)=y_{i}(t)-\alpha_{i} x_{i}(t), i=1,2, \ldots, N$. Subtracting network (4) from (2) gives the error dynamical network

$$
\begin{aligned}
D^{q} e_{i}(t)= & g\left(y_{i}(t)\right)+a \sum_{j=1}^{N} d_{i j} A y_{j}(t)-\alpha_{i} f\left(x_{i}(t)\right) \\
& -\alpha_{i} a \sum_{j=1}^{N} c_{i j} A x_{j}(t)+u_{i}(t), \quad i=1,2, \ldots, N .
\end{aligned}
$$

Then the MPOS problem of drive network (2) and response network (4) is converted into the stability problem of the error dynamical network (11). Our target is to design some appropriate controllers to achieve $\lim _{t \rightarrow+\infty}\left\|e_{i}(t)\right\|=$ $\lim _{t \rightarrow+\infty}\left\|y_{i}(t)-\alpha_{i} x_{i}(t)\right\|=0, i=1,2, \ldots, N$.

\section{The Adaptive Controller for MPOS}

In this section, we will study the MPOS between two FCNs with different topologies or identical topologies by using adaptive control method. We first provide the following main theorem about the MPOS between two FCNs with different topological structure. 
Theorem 7. For the positive definite matrix $A$ in network (2), suppose $T$ satisfies Assumption 6 and let $\theta=\|T\|$. If the following condition is satisfied,

$$
\theta I_{N}+a C-K \leq 0
$$

then the solution $e=0$ of error dynamical network (11) is asymptotically stable under the following controllers:

$$
\begin{aligned}
u_{i}(t)= & \alpha_{i} f\left(x_{i}(t)\right)-g\left(\alpha_{i} x_{i}(t)\right) \\
& -a \sum_{j=1}^{N} b_{i j} A y_{j}(t)-k_{i} A e_{i}(t), \quad i=1,2, \ldots, N,
\end{aligned}
$$

where $I_{N}$ is $N$-dimensional identity matrix, difference matrix $B=\left(b_{i j}\right)_{N \times N}=D-C=\left(d_{i j}-c_{i j}\right)_{N \times N}$, feedback strengths $k_{i}(i=1,2, \ldots, N)$ are positive constants, and constant matrix $K=\operatorname{diag}\left(k_{1}, k_{2}, \ldots, k_{N}\right)$.

Proof. Consider the following $J$ function:

$$
J(t)=\sum_{i=1}^{N} e_{i}^{T}(t) D^{q} e_{i}(t) .
$$

After substituting (11) and (13) into $J(t)$, we have

$$
\begin{aligned}
& J(t)=\sum_{i=1}^{N} e_{i}^{T}(t)\left[g\left(y_{i}(t)\right)-g\left(\alpha_{i} x_{i}(t)\right)\right. \\
& \left.+a \sum_{j=1}^{N} c_{i j} A e_{i}(t)-k_{i} A e_{i}(t)\right] \\
& =\sum_{i=1}^{N} e_{i}^{T}(t)\left[g\left(y_{i}(t)\right)-g\left(\alpha_{i} x_{i}(t)\right)\right] \\
& +a \sum_{i=1}^{N} e_{i}^{T}(t) \sum_{j=1}^{N} c_{i j} A e_{i}(t)-\sum_{i=1}^{N} e_{i}^{T}(t) k_{i} A e_{i}(t) \\
& \leq \sum_{i=1}^{N} e_{i}^{T}(t) T A e_{i}(t)+a \sum_{i=1}^{N} \sum_{j=1}^{N} e_{i}^{T}(t) c_{i j} A e_{i}(t) \\
& -\sum_{i=1}^{N} e_{i}^{T}(t) k_{i} A e_{i}(t) \\
& \leq e^{T}(t)\left[\left(\theta I_{N}+a C-K\right) \otimes A\right] e(t),
\end{aligned}
$$

where $e(t)=\left[e_{1}^{T}(t), e_{2}^{T}(t), \ldots, e_{N}^{T}(t)\right]^{T}$ and $K=\operatorname{diag}\left(k_{1}\right.$, $\left.k_{2}, \ldots, k_{N}\right)$. Because $A>0$, it is easy to conclude that, if (12) is satisfied, the solution $e=0$ of error dynamical network (11) is asymptotically stable.

The proof is completed.

From Theorem 7, we can conclude that as long as feedback strengths $k_{i}$ in feedback matrix $K$ are big enough, the MPOS between network (2) and network (4) can be achieved.

As a special case of different topological structure, MOPS between two FCNs with identical topology can be achieved under the similar adaptive control. In this case, we have $C=$ $D$ and the error dynamical systems become

$$
\begin{aligned}
D^{q} e_{i}(t)= & g\left(y_{i}(t)\right)-\alpha_{i} f\left(x_{i}(t)\right) \\
& +a \sum_{j=1}^{N} c_{i j} A\left(y_{j}(t)-\alpha_{i} x_{j}(t)\right) \\
& +u_{i}(t), \quad i=1,2, \ldots, N .
\end{aligned}
$$

Based on Theorem 7, we can easily obtain the following corollary.

Corollary 8. For two FCNs with identical topology, suppose $T$ satisfies Assumption 6 and let $\theta=\|T\|$. If condition (12) is satisfied, then the solution $e=0$ of error dynamical network (16) is asymptotically stable under the following controllers:

$$
\begin{array}{r}
u_{i}(t)=\alpha_{i} f\left(x_{i}(t)\right)-g\left(\alpha_{i} x_{i}(t)\right)-k_{i} A e_{i}(t), \\
i=1,2, \ldots, N .
\end{array}
$$

Proof. When the topological structures of FCN (2) and (4) are the same, that is, $C=D$, difference matrix $B=\left(b_{i j}\right)_{N \times N}$ becomes a zero matrix. Therefore, the controllers in (13) turn into the controllers described by (17). While substituting (16) and (17) into $J$ function described by (14), the rest of the proof process is the same as that of Theorem 7.

\section{Numerical Simulations}

In this section, illustrative examples are given to verify the effectiveness of the theoretical criteria obtained in the preceding section. Some further analyses about the influence of fractional order $q$ and feedback strength $k_{i}$ on MPOS are provided. For this purpose, we consider fractional order Chen system as the node of drive network and consider fractional order financial system as the node of response network.

4.1. The Verification of Theorem 7 . The well-known fractional order Chen chaotic system [27] is described by

$$
\begin{gathered}
D^{q} x_{1}=a\left(x_{2}-x_{1}\right), \\
D^{q} x_{2}=(c-a) x_{1}-x_{1} x_{3}+c x_{2}, \\
D^{q} x_{3}=x_{1} x_{2}-b x_{3} .
\end{gathered}
$$

When fractional order $q$ holds $q \in[0.7,1]$ and parameters are selected as $a=35, b=3$, and $c=28$, the fractional order Chen system behaves chaotically. Figure 1 displays the attractor of the fractional order Chen chaotic system with $q=0.96$.

Recently, a dynamic model of finance was reported in [28]. The time variation of interest rate $y_{1}$, investment demand $y_{2}$, and price index $y_{3}$ is described in the model. The fractional order financial chaotic system is given by

$$
\begin{gathered}
D^{q} y_{1}=y_{3}+\left(y_{2}-a\right) y_{1}, \\
D^{q} y_{2}=1-b y_{2}-y_{1}^{2}, \\
D^{q} y_{3}=-y_{1}-c y_{3} .
\end{gathered}
$$






FIGURE 1: The chaotic attractor of fractional order Chen system with $q=0.96$.

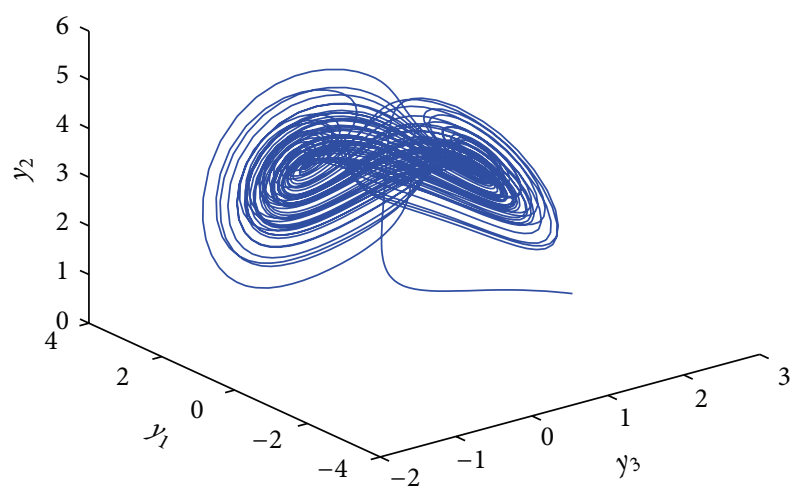

FIgURE 2: The chaotic attractor of fractional order financial system with $q=0.96$.

The fractional order financial system is chaotic when fractional order $q$ changes in $[0.85,1]$ and parameters are selected as $a=3, b=0.1$, and $c=1$. The attractor of the fractional order financial chaotic system with $q=0.96$ is shown in Figure 2.

In the following, we will take the fractional order Chen chaotic system and the fractional order financial chaotic system as node dynamics to illustrate the proposed method for the MPOS. In all the simulations, let the fractional orders of the drive and the response networks be $q=0.96$. And a predictor-corrector scheme [29] is chosen to solve the fractional order differential equations. The network size is taken as $N=5$. Choose the coupling configuration matrices of network (2) and (4) as follows:

$$
\begin{aligned}
C & =\left(\begin{array}{ccccc}
-3 & 0 & 1 & 1 & 1 \\
0 & -2 & 0 & 1 & 1 \\
1 & 0 & -2 & 1 & 0 \\
1 & 1 & 1 & -3 & 0 \\
1 & 1 & 0 & 0 & -2
\end{array}\right), \\
D & =\left(\begin{array}{ccccc}
-4 & 1 & 1 & 1 & 1 \\
1 & -3 & 1 & 1 & 0 \\
1 & 1 & -2 & 0 & 0 \\
1 & 1 & 0 & -2 & 0 \\
1 & 0 & 0 & 0 & -1
\end{array}\right)
\end{aligned}
$$

For simplicity, the inner-coupling matrix is chosen as $A=I_{3}$. The initial values of the state vectors $x_{i}(0)$ and $y_{i}(0)(i=1,2, \ldots, N)$ are chosen arbitrarily in $(-10,10)$. The scaling factor matrix is chosen as $\alpha=\operatorname{diag}(1,-2,3,2,-1)$. The MPOS errors between two networks are defined as $e_{i n}(t)=$ $y_{i n}(t)-\alpha_{i} x_{i n}(t)(i=1,2, \ldots, N ; n=1,2,3)$.

According to Theorem 7, condition (12) is only a sufficient condition for the MPOS between complex networks (2) and (4). Here, we give a choice of feedback matrix $K=\operatorname{diag}(20,15,5,16,10)$ for realizing the MPOS, whose simulation results are shown in Figure 3. As seen in Figure 3, the trajectories of the synchronization errors $e_{i n}(i=$ $1,2, \ldots, 10 ; n=1,2,3)$ approach zero, which imply that the MPOS between networks (2) and (4) has been achieved with the adaptive controllers (13).

4.2. Further Analyses. Research results suggest that the values of fractional order $q$ and feedback strength $k_{i}$ have a great influence on the synchronization effect. Some further analyses about synchronization effect are presented in this section.

Here, in order to know the variations of all the MPOS errors, the norm of the total MPOS errors is defined by

$$
E(t)=\sqrt{\sum_{i=1}^{N} \frac{\left[e_{i 1}^{2}(t)+e_{i 2}^{2}(t)+e_{i 3}^{2}(t)\right]}{N}} .
$$

It is obvious that, when $E(t)$ no longer increases, two fractional-order networks achieve the MPOS globally.

First, we consider that feedback matrix $K=\operatorname{diag}(20$, $15,5,16,10)$ is invariant and only fractional order $q$ is changed. The values of synchronization error $E(t)$ with the increase of the fractional order $q$ are described in Figure 4. From Figure 4, one can easily find that the synchronization effect becomes better with the increase of fractional order $q$.

Second, let control gain $K$ be changed while fractional order $q$ maintains the same. In order to simplify the problem, let $k_{1}=k_{2}=k_{3}=k_{4}=k_{5}$ in current simulations. Figure 5 describes the time evolutions of MPOS error $E(t)$ with the fractional order $q=0.96$ while the feedback strength $k_{i}$ is increasing from 5 to 35 .

It is seen from Figure 5 that the synchronization effect turns better with the increase of the feedback strength $k_{i}$. Moreover, even if some of the $k_{i}$ decrease to 0 , the MPOS can still be achieved in the case of reducing synchronization speed. When the feedback strengths are selected as $k_{1}=k_{2}=$ $k_{3}=10, k_{4}=k_{5}=0$ and $k_{1}=10, k_{2}=k_{3}=k_{4}=k_{5}=0$, the time evolutions of MPOS error $E(t)$ with the fractional order $q=0.96$ are illustrated in Figure 6 .

As mentioned below, different fractional orders and feedback strengths will lead to different rates towards synchronization. The appropriately large fractional order and feedback strength will lead to a fast outer synchronization. Furthermore, the MPOS can also be realized with some of feedback strengths equal to 0 , although the speed of the synchronization will be much slower. 


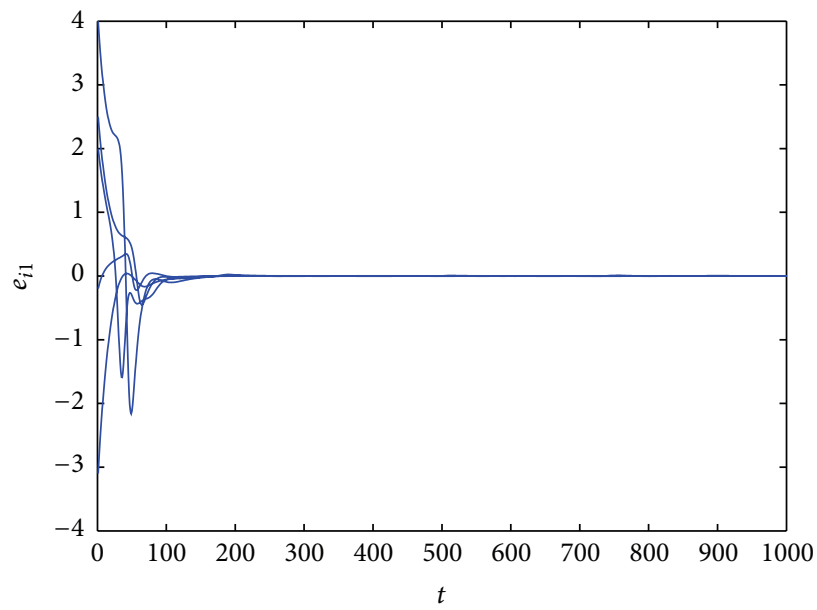

(a)

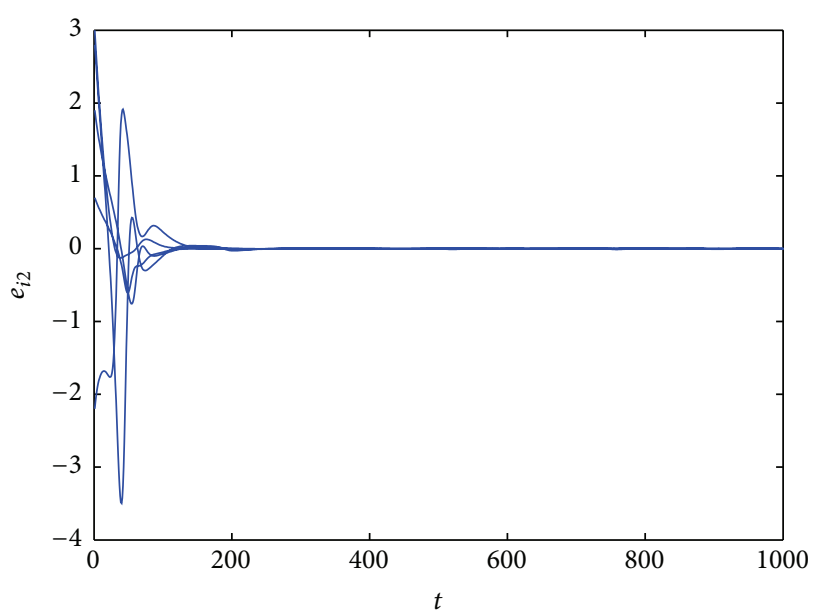

(b)

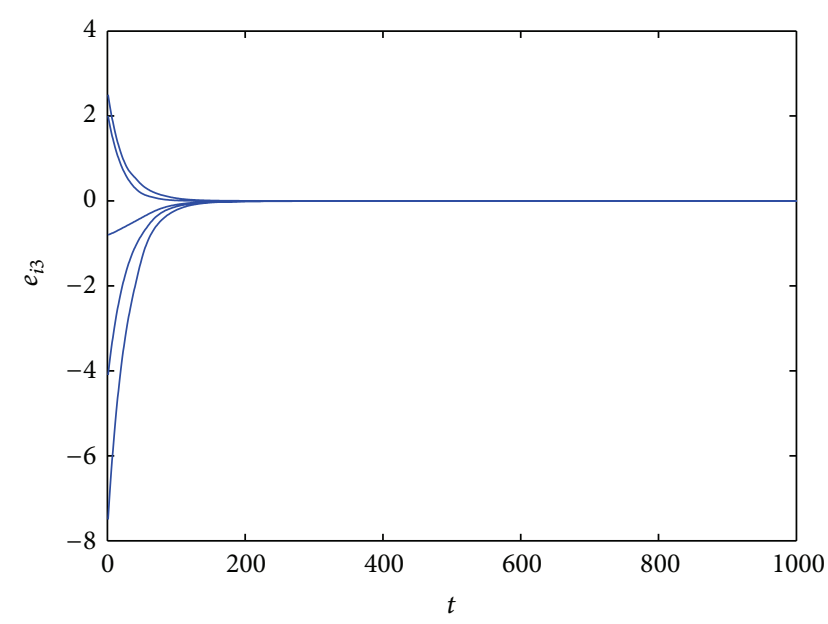

(c)

FIGURE 3: Time evolutions of MPOS synchronization error $e_{i n}$ with time.

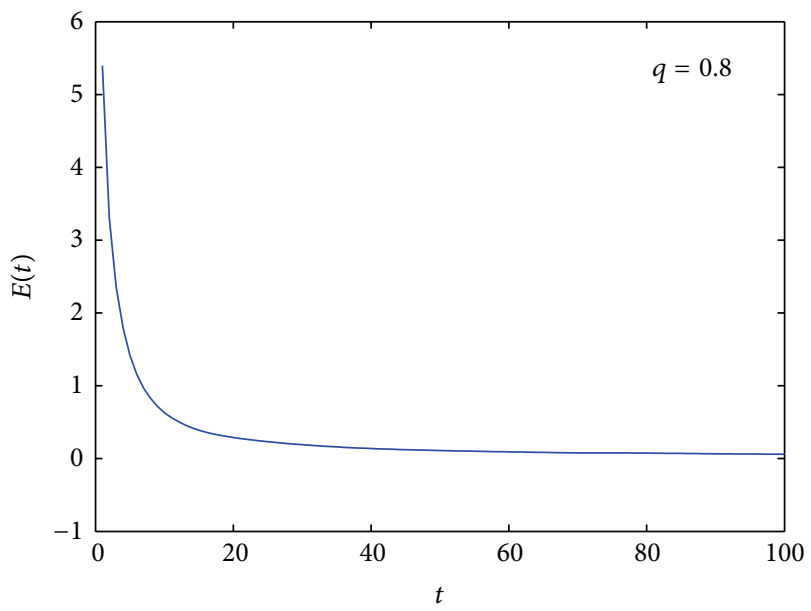

(a)

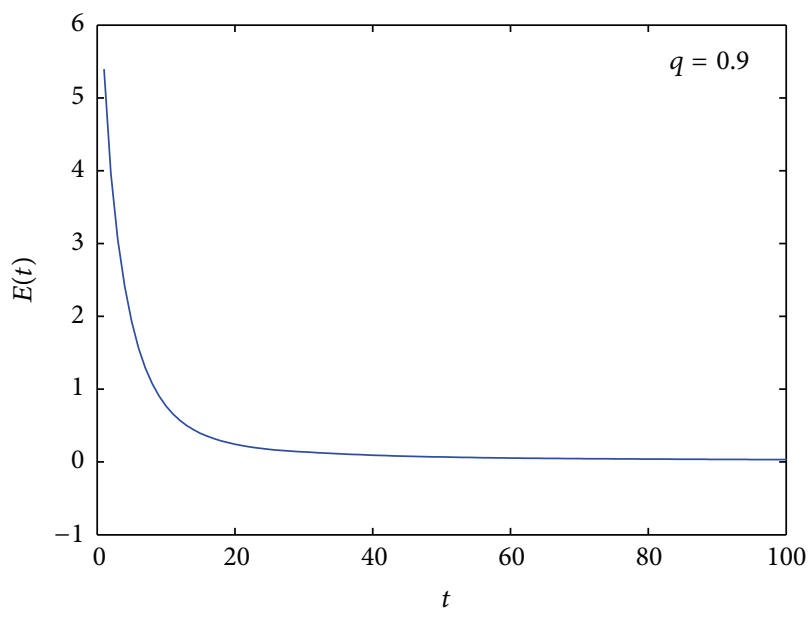

(b)

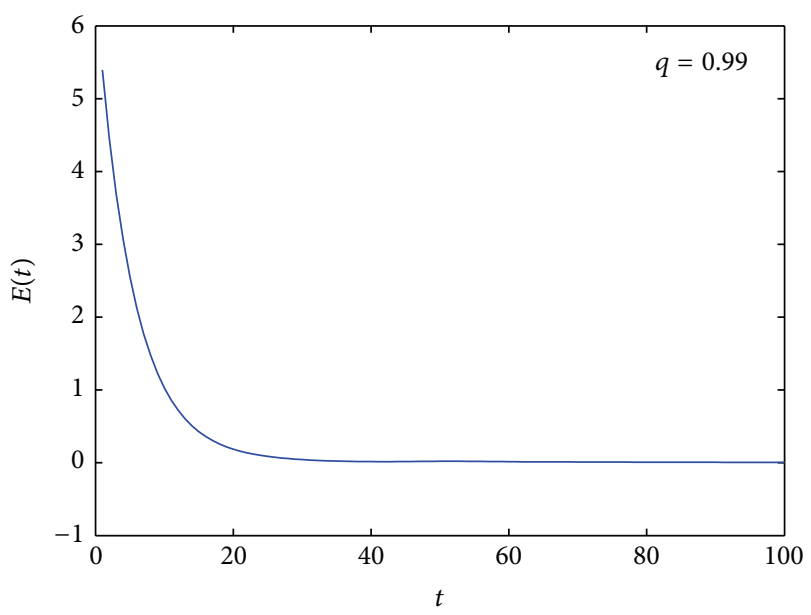

(c)

FIgUre 4: Time evolutions of synchronization error $E(t)$ with different values of fractional order $q$. 


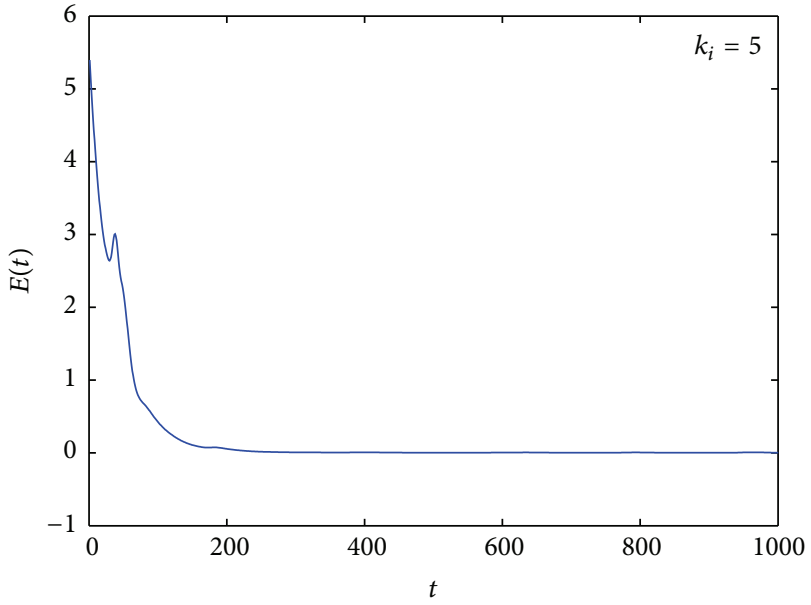

(a)

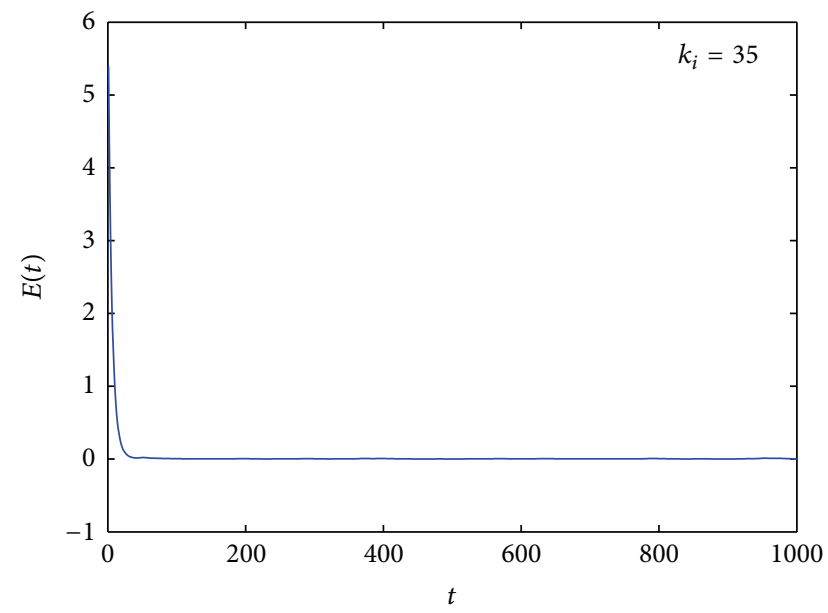

(b)

Figure 5: Time evolutions of synchronization error $E(t)$ with different values of $k_{i}$.

\section{Conclusions}

In summary, the MPOS between two FCNs with different node dynamics and different topological structures is discussed. In the MPOS regime, we can observe different outer synchronization patterns by choosing the scaling factor, for example, complete outer synchronization, antiouter synchronization, and amplitude death. Based on the stability theory of the fractional order system and using the nonlinear control coupling schemes, some sufficient criteria are obtained and the suitable controllers are designed. The sufficient criteria and controllers are also applicable to the case with the same topological structures. This technique has been successfully applied to two fractional order complex networks, which consist of five fractional order Chen chaotic systems and five fractional order financial chaotic systems. Numerical evidence shows that both the feedback strength and the fractional order can be chosen appropriately to adjust the synchronization effect effectively. The larger the fractional order and the feedback strength are, the faster

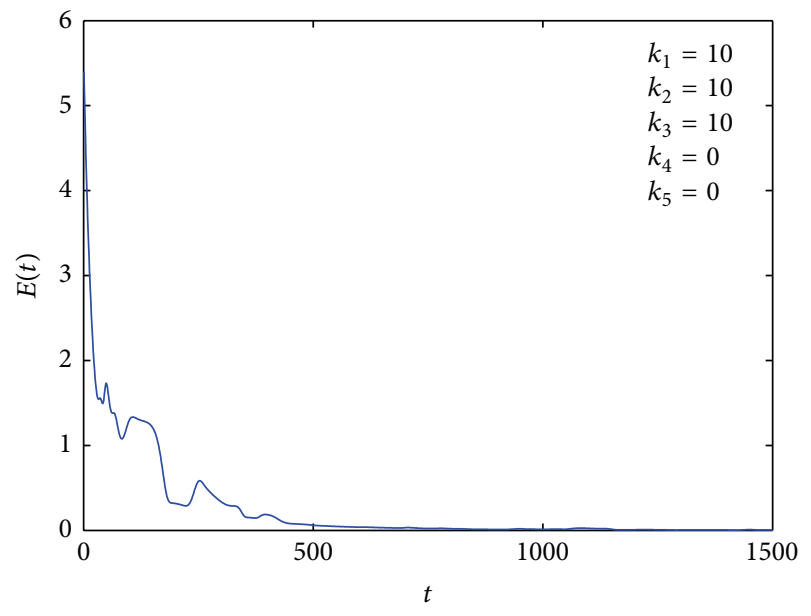

(a)

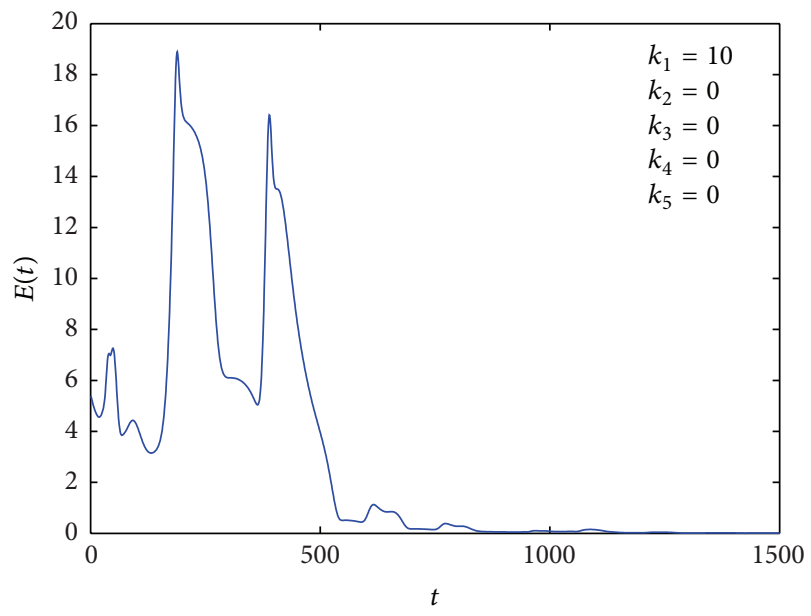

(b)

FIGURE 6: Time evolutions of synchronization error $E(t)$ with some of $k_{i}$ equal to 0 .

the outer synchronization will be achieved. The method can be extended to the condition that the fractional orders of chaotic system in drive networks and response networks are nonidentical or chaotic systems of complex network are with incommensurate orders.

\section{Conflict of Interests}

The authors declare that there is no conflict of interests regarding the publication of this paper.

\section{Acknowledgments}

This research was supported by the National Natural Science Foundation of China (Grant nos. 61374178 and 61202085), the Liaoning Provincial Natural Science Foundation of China (Grant no. 201202076), the Specialized Research Fund for the Doctoral Program of Higher Education (Grant no. 20120042120010), and the Ph.D. Start-up Foundation of 
Liaoning Province, China (Grant nos. 20111001, 20121001, and 20121002).

\section{References}

[1] L. M. Pecora and T. L. Carroll, "Synchronization in chaotic systems," Physical Review Letters, vol. 64, no. 8, pp. 821-824, 1990.

[2] J. Cao, D. W. C. Ho, and Y. Yang, "Projective synchronization of a class of delayed chaotic systems via impulsive control," Physics Letters A: General, Atomic and Solid State Physics, vol. 373, no. 35, pp. 3128-3133, 2009.

[3] G. Z. Feng and J. D. Cao, "Master-slave synchronization of chaotic systems with a modified impulsive controller," Advances in Difference Equations, vol. 2013, article 24, 2013.

[4] J. Lu and J. Cao, "Adaptive complete synchronization of two identical or different chaotic (hyperchaotic) systems with fully unknown parameters," Chaos, vol. 15, no. 4, Article ID 043901, 2005.

[5] D. J. Watts and S. H. Strogatz, "Collective dynamics of "smallworld” networks," Nature, vol. 393, no. 6684, pp. 440-442, 1998.

[6] S. H. Strogatz, "Exploring complex networks," Nature, vol. 410, no. 6825, pp. 268-276, 2001.

[7] A.-L. Barabási, R. Albert, and H. Jeong, "Mean-field theory for scale-free random networks," Physica A: Statistical Mechanics and its Applications, vol. 272, no. 1, pp. 173-187, 1999.

[8] M. E. J. Newman, "The structure and function of complex networks," SIAM Review, vol. 45, no. 2, pp. 167-256, 2003.

[9] X. S. Yang, J. D. Cao, and Z. C. Yang, "Synchronization of coupled reaction-diffusion neural networks with time-varying delays via pinning-impulsive controller," SIAM Journal on Control and Optimization, vol. 51, no. 5, pp. 3486-3510, 2013.

[10] Q. Zhang, J. Lu, J. Lu, and C. K. Tse, "Adaptive feedback synchronization of a general complex dynamical network with delayed nodes," IEEE Transactions on Circuits and Systems II: Express Briefs, vol. 55, no. 2, pp. 183-187, 2008.

[11] W. Yu, G. Chen, and J. Lü, "On pinning synchronization of complex dynamical networks," Automatica, vol. 45, no. 2, pp. 429-435, 2009.

[12] X. Yang, J. Cao, and J. Lu, "Stochastic synchronization of complex networks with nonidentical nodes via hybrid adaptive and impulsive control," IEEE Transactions on Circuits and Systems I: Regular Papers, vol. 59, no. 2, pp. 371-384, 2012.

[13] J. Wang and Y. Zhang, "Network synchronization in a population of star-coupled fractional nonlinear oscillators," Physics Letters A: General, Atomic and Solid State Physics, vol. 374, no. 13-14, pp. 1464-1468, 2010.

[14] L. P. Chen, Y. Chai, R. C. Wu et al., "Cluster synchronization in fractional-order complex dynamical networks," Physics Letters A, vol. 376, pp. 2381-2388, 2012.

[15] M. J. Wang, X. Y. Wang, and Y. J. Niu, "Projective synchronization of a complex network with different fractional order chaos nodes," Chinese Physics B, vol. 20, no. 1, Article ID 010508, 2011.

[16] W. K. Wong, H. J. Li, and S. Y. Leung, "Robust synchronization of fractional-order complex dynamical networks with parametric uncertainties," Communications in Nonlinear Science and Numerical Simulation, vol. 17, pp. 4877-4890, 2012.

[17] G. Wang, J. Cao, and J. Lu, "Outer synchronization between two nonidentical networks with circumstance noise," Physica A: Statistical Mechanics and its Applications, vol. 389, no. 7, pp. 1480-1488, 2008.
[18] Z. Li and X. Xue, "Outer synchronization of coupled networks using arbitrary coupling strength," Chaos, vol. 20, no. 2, Article ID 023106, 2010.

[19] X. Wu, W. X. Zheng, and J. Zhou, "Generalized outer synchronization between complex dynamical networks," Chaos, vol. 19, no. 1, Article ID 013109, 2009.

[20] X. J. Wu and H. T. Lu, "Outer synchronization between two different fractional-order general complex dynamical networks," Chinese Physics B, vol. 19, no. 7, Article ID 070511, 2010.

[21] M. M. Asheghan, J. Míguez, M. T. Hamidi-Beheshti, and M. S. Tavazoei, "Robust outer synchronization between two complex networks with fractional order dynamics," Chaos, vol. 21, no. 3, Article ID 033121, 2011.

[22] M. C. Zhao and J. W. Wang, "Outer synchronization between fractional-order complex networks: a non-fragile observerbased control scheme," Entropy, vol. 15, pp. 1357-1374, 2013.

[23] J. W. Wang and Y. Zhang, "Robust projective outer synchronization of coupled uncertain fractional-order complex networks," Central European Journal of Physics, vol. 11, no. 6, pp. 813-823, 2013.

[24] M. Caputo, "Linear models of dissipation whose Q is almost frequency independent-II," Geophysical Journal of the Royal Astronomical Society, vol. 13, no. 5, pp. 529-539, 1967.

[25] D. Matignon, "Stability results of fractional differential equations with applications to control process," in Proceedings of the IEEE-SMC International Association for Mathematics and Computers in Simulation, pp. 963-968, Lille, France, 1996.

[26] J. B. Hu, Y. Han, and L. D. Zhao, "A novel stablility theorem for fractional systems and its applying in synchronizing fractional chaotic system based on back-stepping approach," Acta Physica Sinica, vol. 58, no. 4, pp. 2235-2239, 2009.

[27] C. Li and G. Chen, "Chaos in the fractional order Chen system and its control," Chaos, Solitons and Fractals, vol. 22, no. 3, pp. 549-554, 2004.

[28] W. C. Chen, "Nonlinear dynamics and chaos in a fractionalorder financial system," Chaos, Solitons and Fractals, vol. 36, no. 5, pp. 1305-1314, 2008.

[29] K. Diethelm, N. J. Ford, and A. D. Freed, "A predictor-corrector approach for the numerical solution of fractional differential equations," Nonlinear Dynamics, vol. 29, no. 1-4, pp. 3-22, 2002. 


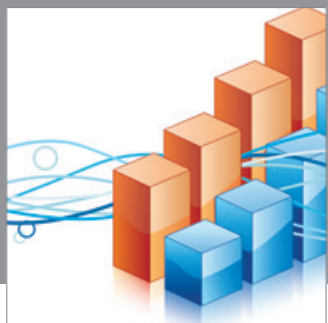

Advances in

Operations Research

mansans

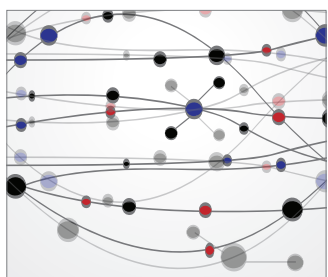

The Scientific World Journal
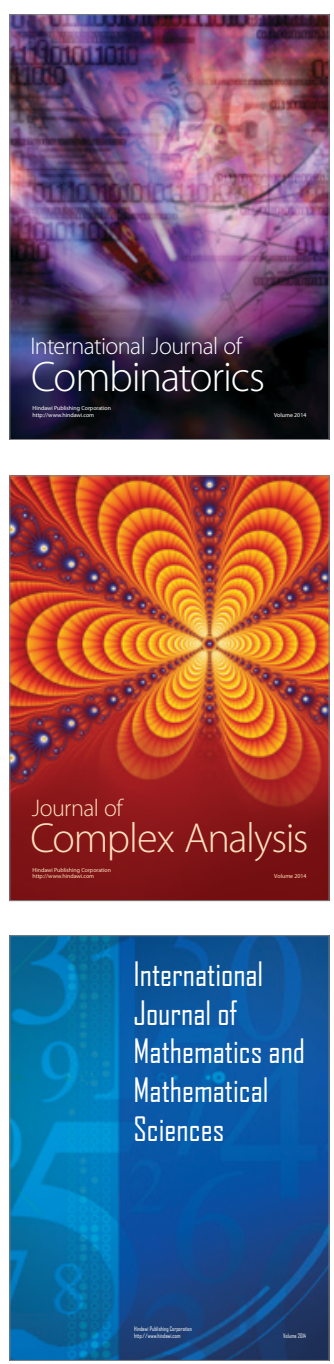
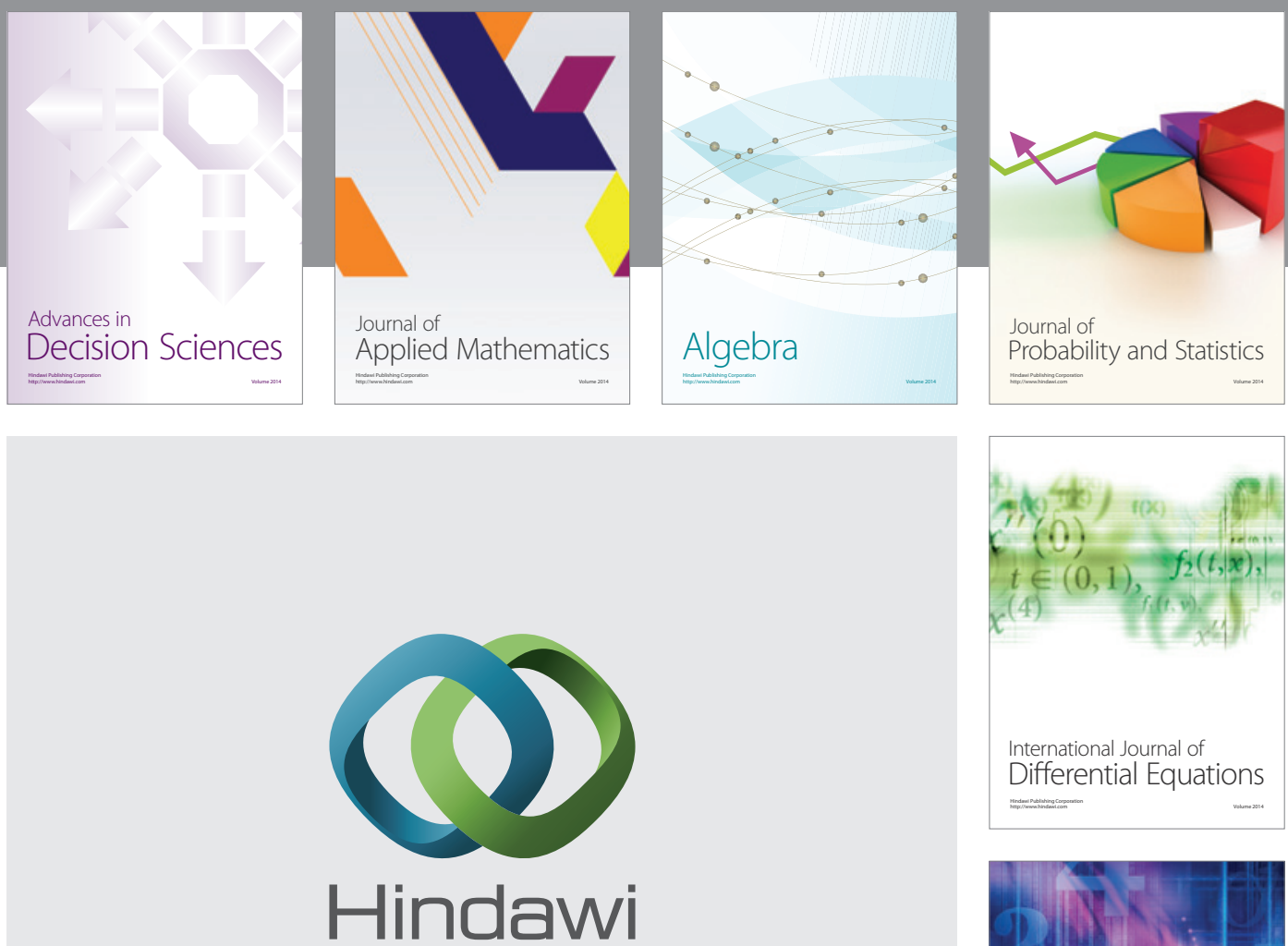

Submit your manuscripts at http://www.hindawi.com
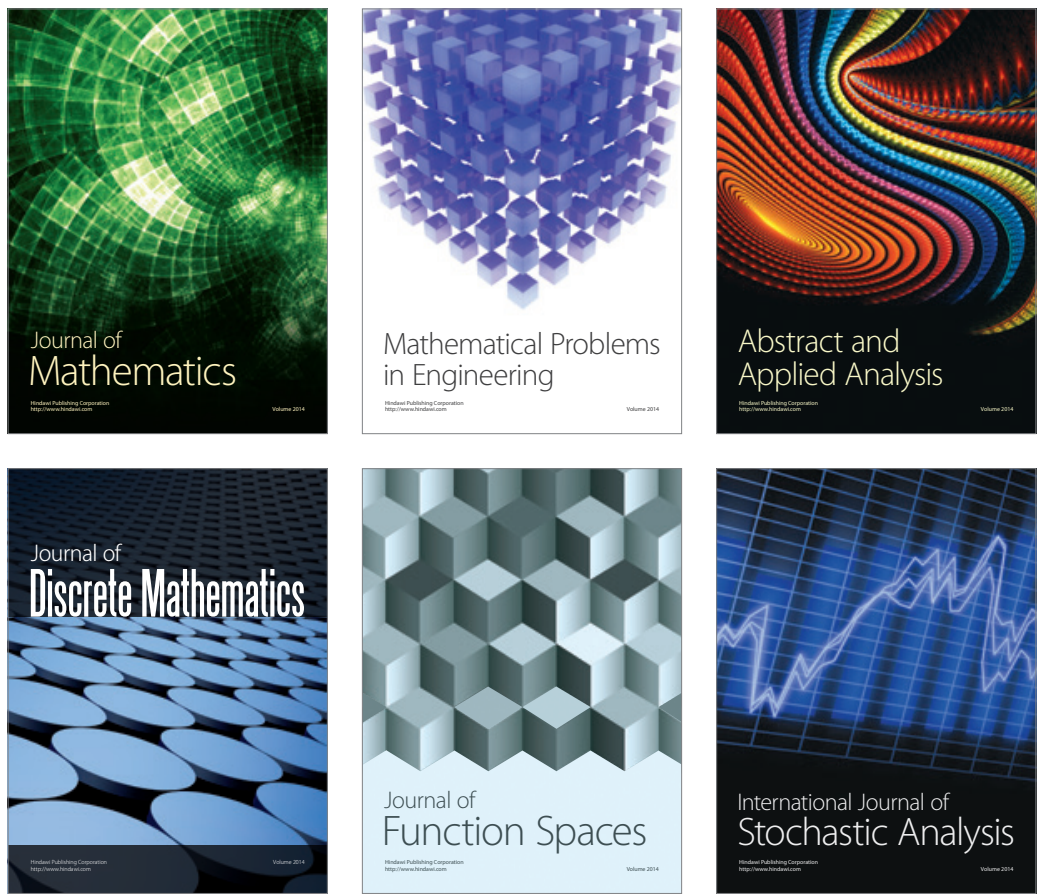

Journal of

Function Spaces

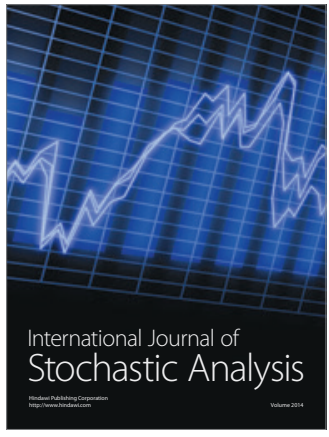

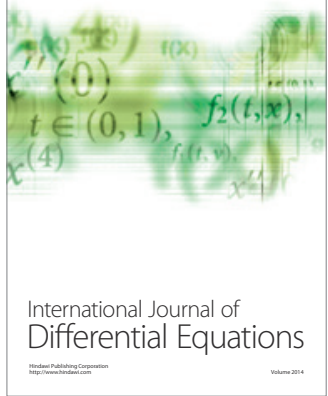
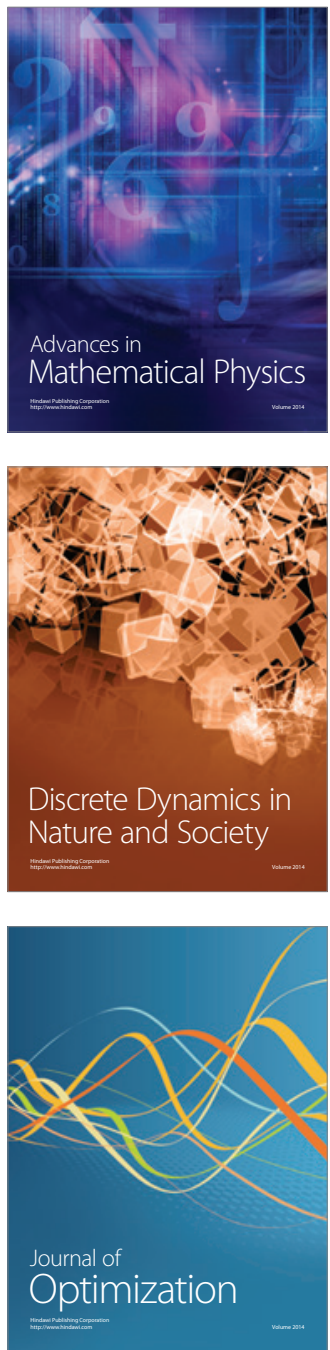